\title{
NATURAL PRODUCTS IN ORGANIC SYNTHESIS
}

\author{
ROBERTH. BURNEL L \\ Departement de chimie, Universite Laval, Quebec \\ Quebec, Canada GIK 7P4
}

\begin{abstract}
A resume of four lectures presented by the author during a one month visit to BIOTROP in 1995. The seminars were held at the University of Indonesia at Depok, the Technical Institute in Bandung, the Bogor Agricultural Institute and at BIOTROP, Bogor. The talks were grouped under the general title "The Use of Natural Products in Organic Synthesis".
\end{abstract}

Key words: Organic compounds/Natural products.

The venerable art of the chemical synthesis of naturally occurring organic compounds which has inspired and fascinated chemists for roughly one hundred and fifty years continues to be necessary today for several reasons. First, and traditionally the most important reason, is that synthesis provides the ultimate proof of structure of a substance by showing that it can be prepared in an unambiguous fashion from simple building blocks. In principle the structures of all the hundreds of new compounds isolated from Nature every year should be synthesized. However, if new substances are very similar in structure they can be interrelated by conversion to one another or perhaps to products that have already been synthesized.

A second reason for practicing this demanding art we call synthesis is the challenging necessity of preparing increasingly complex substances in more efficient ways and this is the most important source of innovation and invention that assures progress in the organic field.

Of course, synthesis can also provide an alternate to Nature as the source of useful compounds. For instance, despite the effectiveness of the anti-cancer natural product taxol, it is a sobering thought that all the Taxus trees in the world could not supply at present enough of the drug to treat the women actually suffering from breast and cervical cancer. And to make matters worse, the trees take almost a century to mature. Furthermore it has been estimated that by the year 2000 there will be a world wide short fall of several tons of the natural anti-malarial obtained from Artemesia species. The difficulty of shortages can sometimes be alleviated by synthesizing simpler and cheaper analogs. 
(Burnell et al. 1985), maytenoquinone (Burnell et al. 1988) and coleon B (Burnell et al. 1983), while occasionally we were able to correct the published structures, as in the case of the nellionols (Burnell et al. 1984).

For structure confirmation, the simpler synthesis of the left and right handed mixture (the racemic substance) is quite adequate because as mentioned, with the exception of the optical property of rotating the plane of light, the left hand form, the right hand form or the 50:50 mixture of the two, all show the same spectroscopic behavior and these are the characteristics we use to identify them (ultra-violet, infra-red, nuclear magnetic resonance and mass spectra).

Strictly for proofs of structure, we have prepared molecules by total synthesis from smaller symmetrical, easy to buy or make, starting materials (Burnell et al. 1993). The general strategy is shown in the upper part of Scheme 6. To the rather commonplace aromatic compound 30 we attached a long chain of carbons derived from natural geraniol 29. This flexible substituent has two "double" bonds in critical positions enabling it to form two rings when attacked by a suitable "positively charged" reagent $\left(\mathrm{Ph}-\mathrm{S}^{+}\right.$in our case). Both the $\mathrm{Ph}-\mathrm{S}$ and the $\mathrm{CN}$ groups in the product 32 can be exchanged by oxygen and this completed a rapid synthesis of methyl nimbinone 33, a diterpenoid from the "Neem" tree, Azadirachta indica (Ira et al. 1988 (1)).

The final product from the rapid synthesis was "racemic", a 50:50 mixture of the natural substance and its mirror image and all the physical properties of our sample (except the rotation of light) were identical with those in the literature. Obviously the proposed structure is correct! To compare the two approaches we also prepared methyl nimbinone from natural podocarpic acid (Burnell et al. 1993). This far more arduous route is summarized in the lower part of Scheme 6. The key step in this sequence was the formation and subsequent opening of the three-membered ring as a method of installing the two methyl groups on the first ring of the skeleton. In this case when the synthesized methyl nimbinone 36 was examined in the polarimeter, it did rotate the plane of polarized light in the same direction and to exactly the same extent as the product isolated from Nature. This synthesis not only confirms the general structure forwarded for the diterpene, it also tells us that the substance elaborated by the Neem tree is precisely and exclusively as represented in formula 36 . That is, if the three rings of the skeleton are in the plane of the paper, the methyl group identified by the dark wedge between the rings, protrudes towards the reader.

Choosing between the rather simple straight-forward synthesis that leads to a mixture of the two image forms of methyl nimbinone or the more difficult and less rewarding preparation of the real natural diterpene from natural podocarpic acid depends on the real goal of the synthesis. But it would obviously be an enormous advantage to be able to combine the simplicity of the former with the specificity of the latter. To investigate this possiblity we selected another relatively simple compound, methyl nim- 
bionone 44 to experiment with this "enantiomeric" style of synthesis. This diterpene is similar in name and in structure to methyl nimbinone 36 and it was also extracted from the "Neem" tree (Ira et al. 1988(2)). Crucial to this approach is the incorporation of an asymmetric unit (technically a "chiral auxilliary") into the starting material at the outset. In theory, the "left or right-handedness" of this appendage will just be borrowed temporarily during the synthesis and it should impose its asymmetry on the reactions to induce the preferred formation of just one of the mirror image forms of the final product. At a propitious moment this helpful but perhaps costly auxilliary can be removed and recovered to be used in another synthesis.

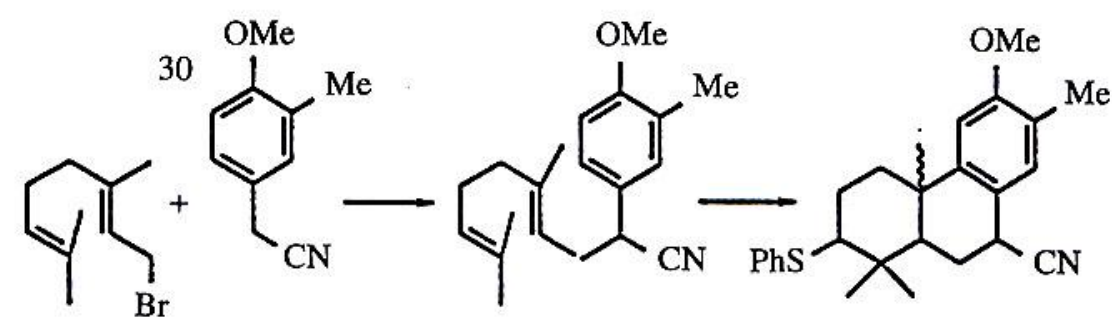<smiles>C[C@]12CCC[C@@](C)(C(=O)O)[C@H]1CCc1ccc(O)cc12</smiles>

31<smiles>COc1cc2c(cc1C)C(C)(C)C1CC(C)(C)C(=O)CCC21C</smiles>

Podocarpic acid

23

Methyl nimbinone 33<smiles>[R20]c1cc2c(cc1C)CCC1C(C)(C)C(O)CC[C@@]21CCC</smiles> 
We chose to attach to the necessary aromatic starting product 43, the asymmetric unit 42 (our auxilliary) invented by Evans (Evans el al. 1985) which we had previously prepared from the abundant natural amino acid, L-valine 41. As in the earlier synthesis, we added the ten carbon bromide 29 and immediately we knew the auxilliary was performing its function. While there are two three-dimensional structures possible for the product 45 , only one of the two was formed in quantity in the reaction!

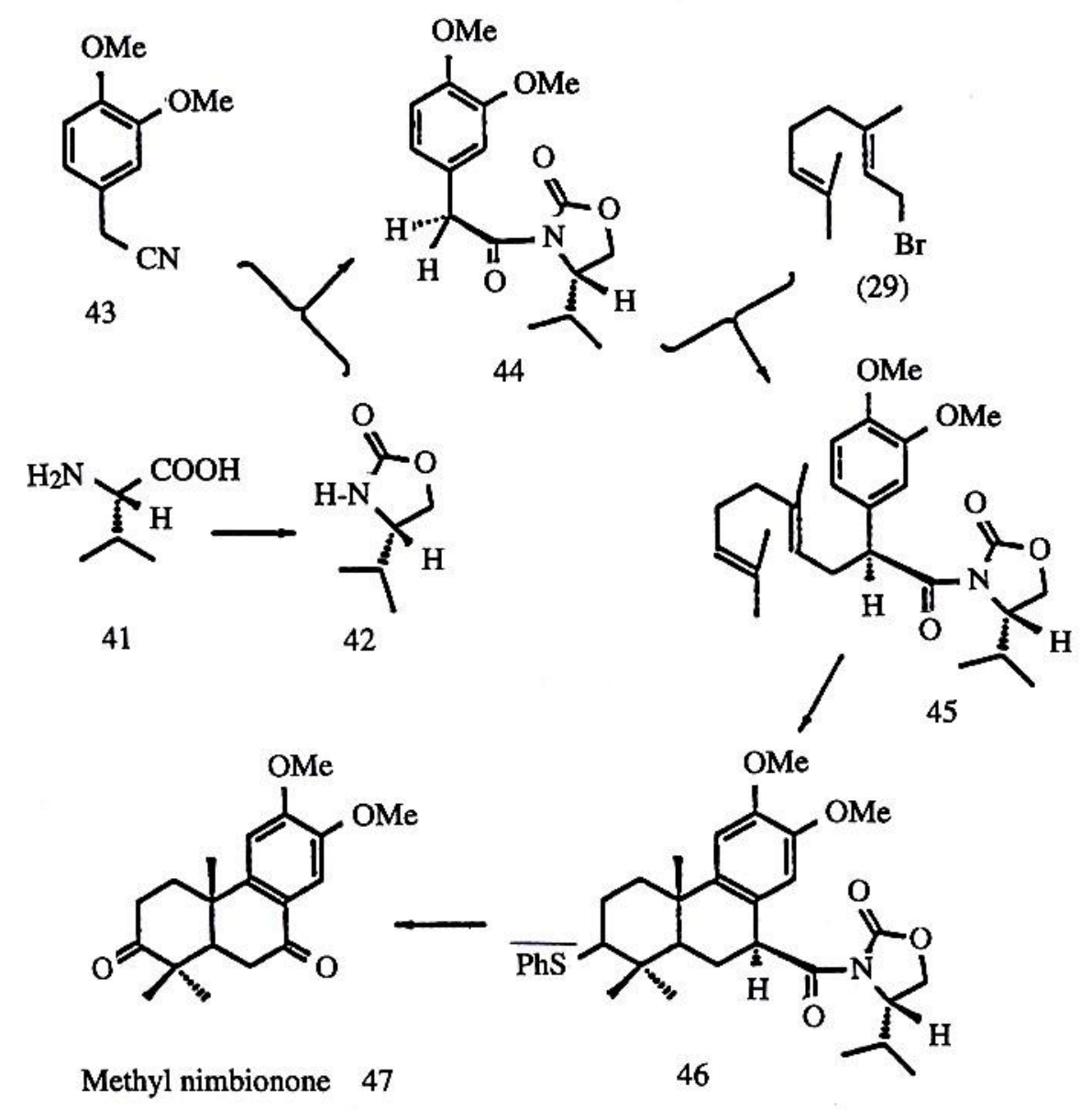


Employing roughly the same steps as in the methyl nimbinone sequence (above), the intermediate 45 was cyclized to compound 46 and the two oxygen functions were introduced to afford a product which showed all the spectroscopic attributes of methyl nimbionone 47 (Dumont 1993). It was encouraging that examination of the optical properties of the product in the polarimeter showed that the substance did indeed rotate the plane of light. However, the magnitude of the rotation was much lower than that recorded for the natural product which indicated that the high "left hand, right hand" selectivity we had achieved early in the synthesis (only one form of 45 was isolated), had been scrambled or lost in the later steps. We suspect that the conditions for the cyclization (45 to 46) are too severe and our efforts are now aimed at finding milder alternate methods of performing this step. The improved conditions we are searching for must provide the product in high yield, sufficient to enable the completion of the synthesis which still involves several steps. Unfortunately, as yet, we have not found this more gentle procedure.

\section{ACKNOWLEDGEMENTS}

The work described was financed partly by NSECC (the Natural Sciences and Engineering Council of Canada), partly by the Government of Quebec (F.C.A.R.) and more recently by generous personal funding (Emeritus Professor Charles R. Engel). My sincere appreciation is extended to the following graduate students whose collaboration and talent were crucial to the study : Sonia Desfosses, Nathalie Dumont, Nathalie The-berge, Michel Jean and David Miller.

The author wishes to thank the staff at BIOTROP for their hospitality (in particular Dr. Hilman Affandi and Dr. Gloria Enriquez) and the Canadian International Development Agency (CIDA) for making the visit to Indonesia possible.

\section{LTERATURE CITED}

BARTON,D.H.R., A.G. BREWSTER, S.V. LEY, C.M. READ and M.N. ROSENFELD. 1981. Oxidation of phenols, pyrocatechols and hydroquinones to orthoquinones using benzeneseleninic anhydride. J. Chem. Soc., Perkin. Trans. I., 1473-1476. BREWSTER, J.H. 1959. A useful model of optical activity. J. Amer. Chem. Soc., 81: 5475-5483.

BURNELL, R.H., A. ANDERSEN, M. NERON-DESBENS and S. SAVARD. 1981. Approaches to the synthesis oflyco-xanthol and similar natural products. Synthesis of coleon U. Can. J. Chem., 59: 2820- 2825.

BURNELL. R.H., M. JEAN and S. SAVARD. 1983. Synthesis of coleon B tetramethyl ether. Can. J. Chem., 61: 2461-2465. 
BURNELL. R.H., M. JEAN, D. POIRIER and S. SAVARD. 1984. The structures of the nellionols. Synthesis of model abieta-8,11,13- trien-7-ones. Synthesis of 5-dehydronellionol trimethyl ether. Can. J. Chera., 62: 2822-2829.

BURNELL, R.H., A. ANDERSEN, M. NERON and S. SAVARD. 1985. Synthesis of coleon C tri-O-methyl ether. Can. J. Chem., 63: 2769-2776.

BURNELL, R.H., M. JEAN and D. POIRIER. 1987. Synthesis of taxodione. Can. J. Chem., 65: 775-781.

BURNELL, R.H., M. JEAN and S. MARCEAU. 1988. Synthesis of maytenoquinone. Can, J. Chem., 66: 227-230.

BURNELL, R.H., N. DUMONT and N. THEBERGE. 1993. Synthesis of O- methyl-nimbinone. J. Nat. Prod., 56: 1930-1936.

CARRIERE, Y., J. MILLAR, J.N. MCNEIL, D.J. MILLER and E.W. UNDERBILL. 1988. Identification of the female sex pheromone in alfalfa blotch leafminer, Agromyzafrontella (Rondani). J. Chem. Ecol., 14: 947-956.

DUMONT, N. 1993. Synthese de diterpenes naturels isoles de 1' Azadirachta indica (Neem). M.Sc. thesis, University Laval, Canada.

EVANS. D.A., D.J. MATHRE and W.L. SCOTT. 1985. Asymmetric synthesis of the enkephalinase inhibitor Thiorphan. J. Org. Chem., 50: 1830-1835.

FURIA, T.E. and N. BELLANCA. 1971. Fenaroli's Handbook of Flavor Ingredients. The Chemical Rubber Co., Cleveland, Ohio.

IRA, B.S., S. SIDDIQUI, S. FAIZI and B.S. SIDDIQUI. 1988 (1). Tricyclic diterpenes from the stem bark of Azadirachta indica. J. Nat. Prod., 51: 1054-1061.

IRA. B.S., S. SIDDIQUI, S. FAIZI and B.S. SIDDIQUI. 1988 (2). Teipenoids from the stem bark of Azadirachta indica. Phytochemistry, 27 : 1801-1804.

KING,F.E., T.J. KING and J.G. TOPLISS. 1956. Synthesis of podocarpic acid. Chem. and Ind., 113.

KUPCHAN, S.M., A. KARIM and C. MARCKS. 1969. Tumor inhibitors. XVIII. Taxodione and taxodone, two novel diterpenoid quinone methide tumor inhibitors from Taxodium distichium. ]. Org. Chem., 34: 3912.

MILLER, D., F. BILODEAU and R.H. BURNELL. 1991. Stereoselective synthesis of isomers of 3,7-dimenthyl-nonadecane, a sex pheromone of the alfalfa blotch leafminer (Agromyza frontella (Rondani)). Can. J. Chem., 69: 1100-1106. 
Yet another motivation for synthesis stems from the fact that Nature very often provides only very small quantities of the substances of the greatest physiological interest. Modern analytical instruments enable the chemist to elucidate the structures of these unknown, potentially useful biological, medicinal or phytochemical compounds even if the elaborate extraction procedures afford only a few milligrams of material. However, for proper assessment of their physiological activities and their mechanisms of action, much greater amounts are probably needed and only synthesis could provide the necessary quantities.

Most of the interesting molecules of Nature are asymmetric rather like our hands. Each hand is made up of the same elements such as fingers, thumbs, palms, nails and so on and while they are very similar, they are not identical. A right-hand glove does not fit on the left hand ! In fact our hands are mirror images of one another. Natural molecules made of the same atoms could be assembled in two fashions, one being the image of the other. Unlike our hands, however, Nature usually synthesizes only one of the two forms (chemically we call the two images "enantiomers"). Since all "our human" metabolic intermediates and enzymes are also asymmetric, the physiological activity of a natural substance may be quite different from that of its mirror image. The "enantiomeric" form could just be inactive or less active than the natural product but it could have harmful effects or be highly toxic. The much quoted case of Thalidomide springs to mind where one enantiomer was a benign sedative recommended for women suffering nausea during pregnancy whereas the mirror image of exactly the same molecule was teratogenic producing babies with serious physical defects. So synthesis must not only aim at preparing the target molecule with the right general structure but the methodology should also lead to the right three-dimensional structure (or stereochemistry). In simpler terms, the synthesis must give exclusively the "left" or exclusively the "right" hand molecule!

Chemically producing this "left or right" hand configuration is extremely difficult. The problem is further compounded by the fact that virtually all the physical properties of the two image forms are identical which means we cannot easily tell them apart and, furthermore, we cannot separate one from the other !! However, two characteristics of the enantiomers (images) are different. First, as we have seen, they can vary greatly in their physiological activities. Secondly, like all asymmetric substances, they rotate the plane of polarized light but the images turn the light in opposite directions. Fortunately we can measure this in a polarimeter and under the conditions defined by the test, a fresh solution of the more prevalent form of the common sugar glucose rotates light through 112 degrees in a clockwise or right-handed fashion while its image would also turn light 112 degrees anti-clockwise or to the left. In fact we often say that a substance such as glucose is dextro-rotatory (from the Latin dextrus : right) or we just abbreviate this to d-glucose where the small "d" shows that light passing through a solution of the product 
would be turned to the right. The image form would be levo-rotatory and called 1-glucose. (Another convention describes rotation to the right as positive (+) and rotation to the left as negative or (-)).

One way of assuring that the molecule targeted in a synthesis will have the right stereochemistry (left or right-handedness) is to start the synthesis from an asymmetric substance available in pure form in Nature. Many potentially useful, commercially available compounds exist from simple amino acids or sugars to complex steroids, alkaloids and terpenes. Our work on an insect pheromone is a simple example of such an "enantio-selective" synthesis (as reaction sequences aimed exclusively at the right or left handed structures are called).

The insect, the small fly Agromyza frontella was accidentally introduced into Canada from Europe in the 1960's and its presence was rapidly detected because in its larval stage it destroyed the leaves of the popular cattle fodder, alfalfa. In their breeding ritual, the female insects attract the males by emanating a minute quantity of a chemical messenger or pheromone and although the quantity of this substance available was infinitesimal, structure 1 (or 3,7-dimethylnonadecane) was advanced for the pheromone. The structure elucidation was made possible by a skillful study of the mass spectral fragmentation after rigorous purification by preparative gas chromatography (Carriere et al. 1988). Potentially this natural chemical could be used to control the population of the insect by using it to divert the male flies away from the females and thus preventing reproduction !

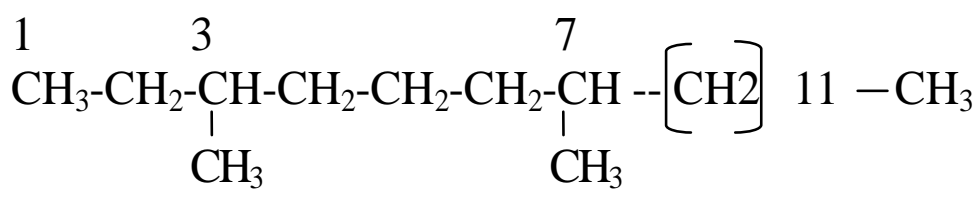

At first glance the substance is chemically uninteresting. It is just a member of the stubbornly unreactive class of organic substances known as paraffins or saturated acyclic hydrocarbons. On reflection, however, the pheromone is a synthetic challenge because the positions of the two methyl groups (CHs or Me groups on the third and seventh carbons of the long chain) make the molecule asymmetric so in fact there are four spatial arrangements possible, as shown in structures 2 to 5 . Only one will be the active pheromone but which one ? Our synthetic strategy should enable us to prepare one (or better all) of them specifically. 


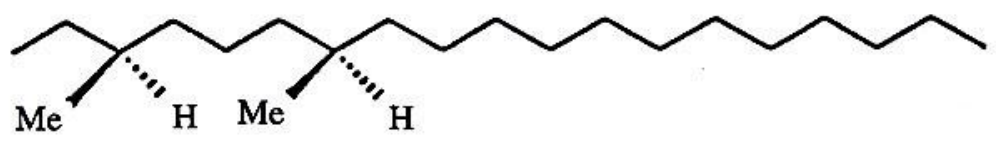

2 Both Me on the near side

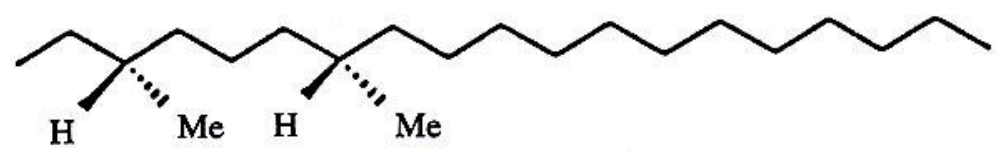

3 Both Me on far side<smiles>CCCCCCCCCCCC[C@H](C)CCC[C@H](C)CC</smiles>

4 One Me up and one down<smiles>CCCCCCCCCCCC[C@H](C)CCC[C@H](C)CC</smiles>

5 Other Me Up and other down

In designing a synthesis we normally work backwards from the target, hypothetically disconnecting parts we feel we can graft back onto the remaining core using chemical methods of proven specificity and geometry. Our first idea for the synthesis of the Agromyza pheromone 1 arose from the "retro-synthesis" shown in Scheme 1. Disconnections leave a central portion which contains both of the methyl branches on the long chain. Many naturally occurring oils (commercially available at low price) contain compounds with this skeleton and our final choice of a "starting material" would be motivated by the need for elements of structure permitting the easy grafting of the other units. And, of most importance, the molecule should be asymmetric with at least one "left" or "right" hand centre. After considering several possibilities, pulegone 6, the main constituent of the "Oil of European Pennyroyal" from Mentha pulegioides L. (Furia and Bellanca 1971) seemed ideal for the purpose. 

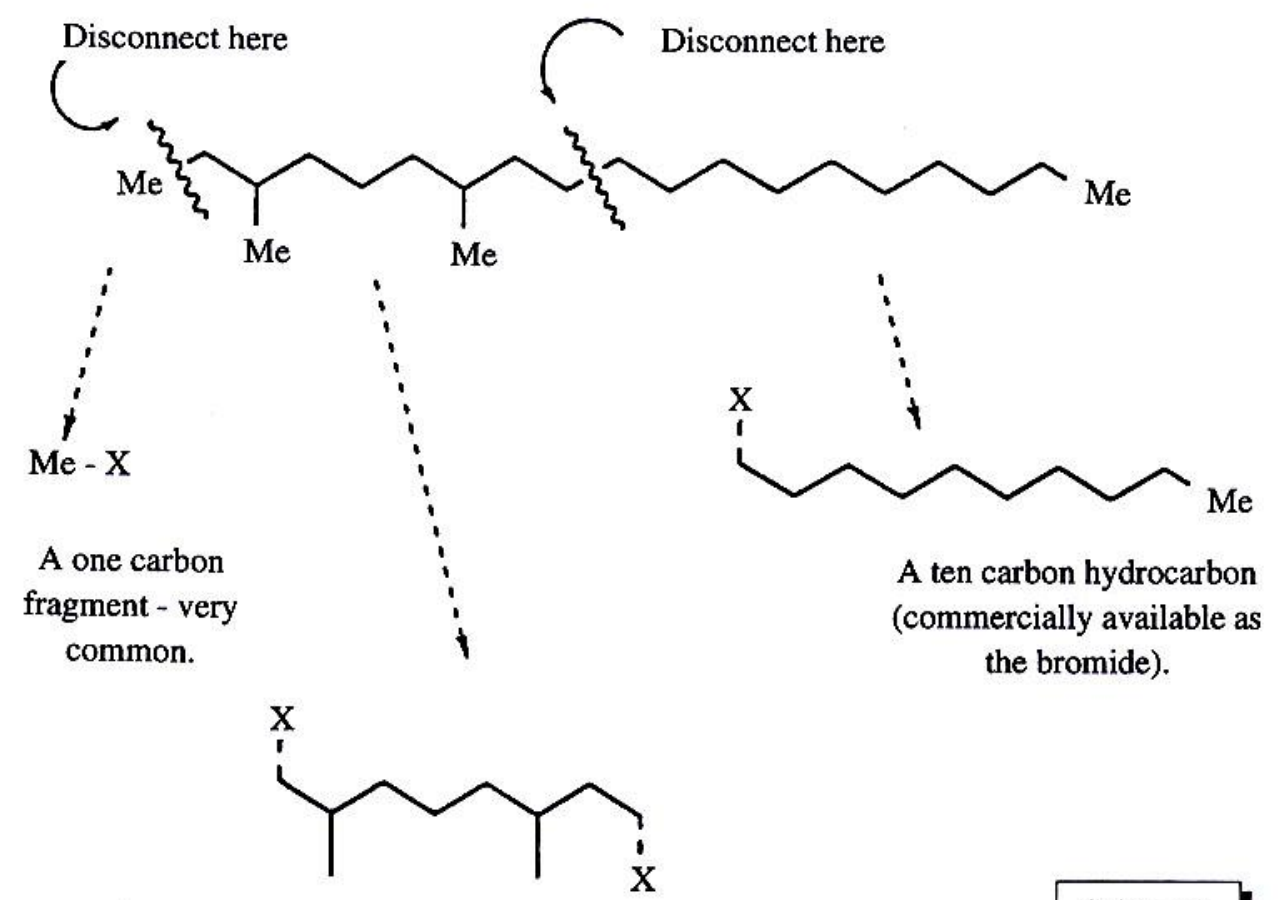

Scheme 1

A central ten carbon unit which has the skeleton of a natural mono-terpene.

Without commenting at length on the chemical reactions involved, we constructed the pheromone as shown in Scheme 2. For those interested in how the transformations were achieved, the reagents (indicated by the small letters on the arrows) are, of course, published in detail (Miller et al. 1991). The steps from 6 to 11 not only add the "one carbon unit" but also induce the stereochemistry (left or right-handedness) at C.3. None of these chemical transformations could have altered the configuration of the methyl substituent of the original pulegone so the geometry at the other methyl branch, now further in the chain at C.7, survives unchanged from beginning to end.

Mechanistic and theoretical arguments told us that the final product from our synthesis was actually structure 3, the drawing of which implies that if the long chain zig-zags in the plane of the paper then both methyl groups represented by the dotted wedges, stick out to the rear. Other considerations told us that of the four possible structures this was actually the active pheromone. When analyzed by polarimetry and using rules formulated by Brewster for calculating the optical properties of hydrocarbons 

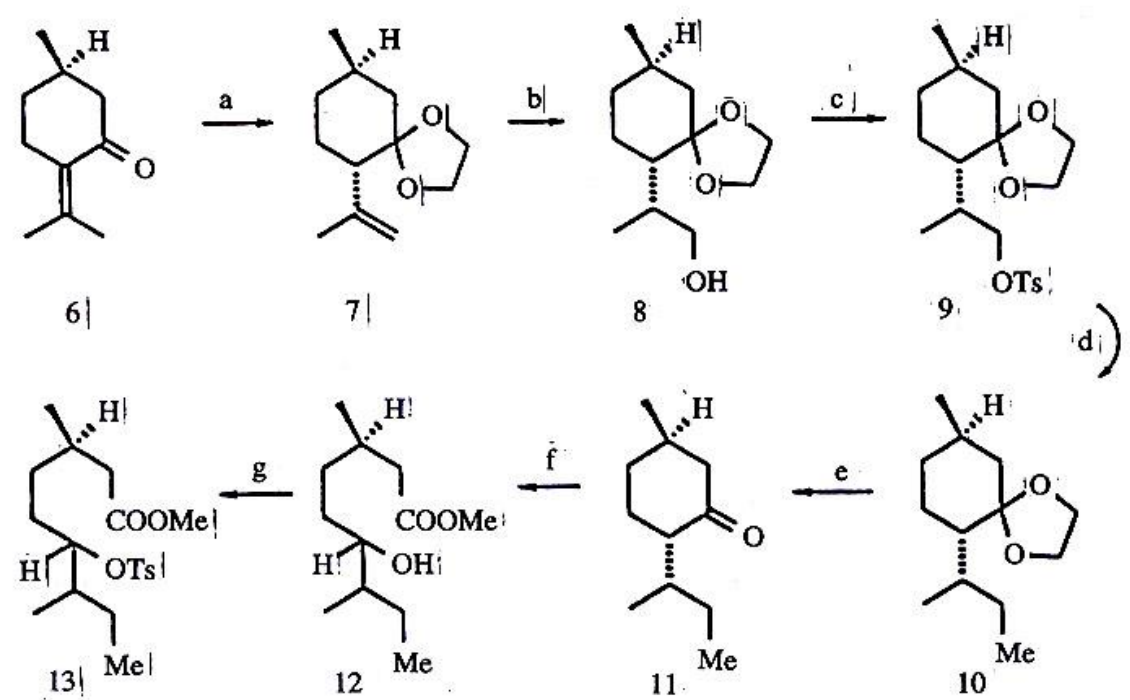

(h)
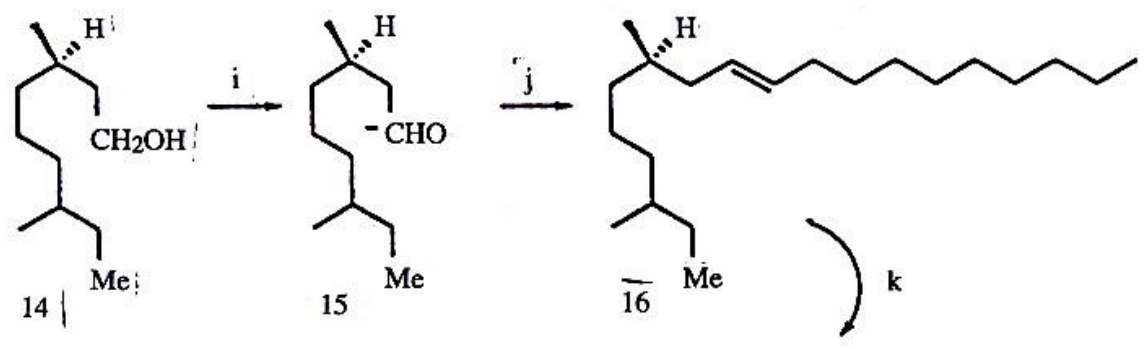

Scheme 2

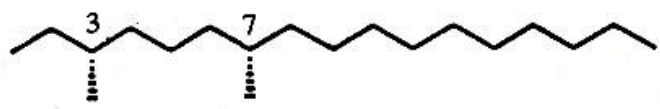

Pheromone : structure 3

(Brewster 1959), we felt the synthetic oil also contained lesser quantities of structure 4 in which the C.3 methyl has the other configuration. 


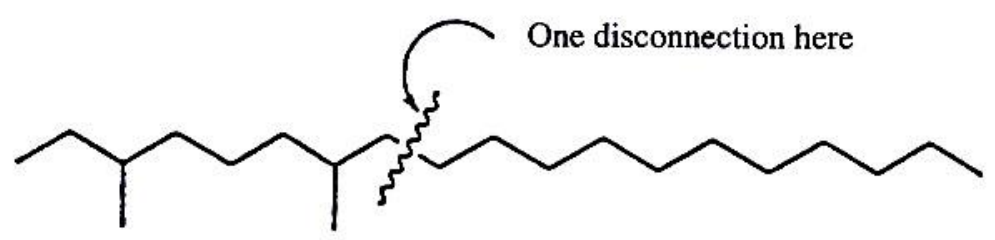

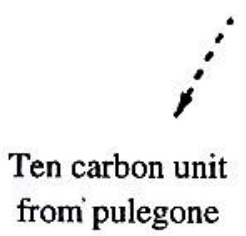

from pulegone
Scheme 3

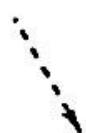

An eleven carbon chain (commercially available)

We had chosen pulegone as the starting material for the synthesis for another reason. If the disconnetion suggesting possible synthetic approaches is envisaged in another fashion as shown in Scheme 3, the pheromone could be prepared by just grafting an eleven carbon fragment onto the pulegone 6 .

So, to check both the structure and the purity of the end product from our first synthesis, we prepared the pheromone a second time by this alternate route which as shown in Scheme 4, starts from compound 8, an early intermediate in the previous synthesis (Miller $e t$ al. 1991).

This second sequence gave exactly the same pheromone 3 but in higher yield. Remarkably in this case and as intended, the original asymmetry of pulegone (the methyl branch) is now at C.3 rather than at C.I as in the first synthesis. This "left hand : right hand" selection was only possible because the natural pulegone is asymmetric and the Pennyroyal plant synthesizes only one of the two possible mirror image forms. Interestingly, the image form of pulegone can be isolated in pure form from other plant species so our synthesis could also lead to the image of pheromone 3 represented by structure 2 .

A second example of synthesis in our laboratory starting from commercially available, naturally occurring asymmetric substances involves Podocarpic acid 23 which was itself synthesized as early as 1956 (King et al. 1956). The world source of this acid is the resin obtained from Podocarpus trees found here in Java and New Zealand. One of the molecules we hoped to synthesize was Taxodione, an anti-tumoral substance shown to possess structure 28 (Kupchan et al. 1969) and at that time was considered a promising drug for human therapy. When we started, other syntheses had already been reported by other groups but we felt that we could improve on existing syntheses in elegance and efficiency. Podocarpic acid was a natural choice as starting material because even uninformed inspection shows that it already possesses many of the structural 


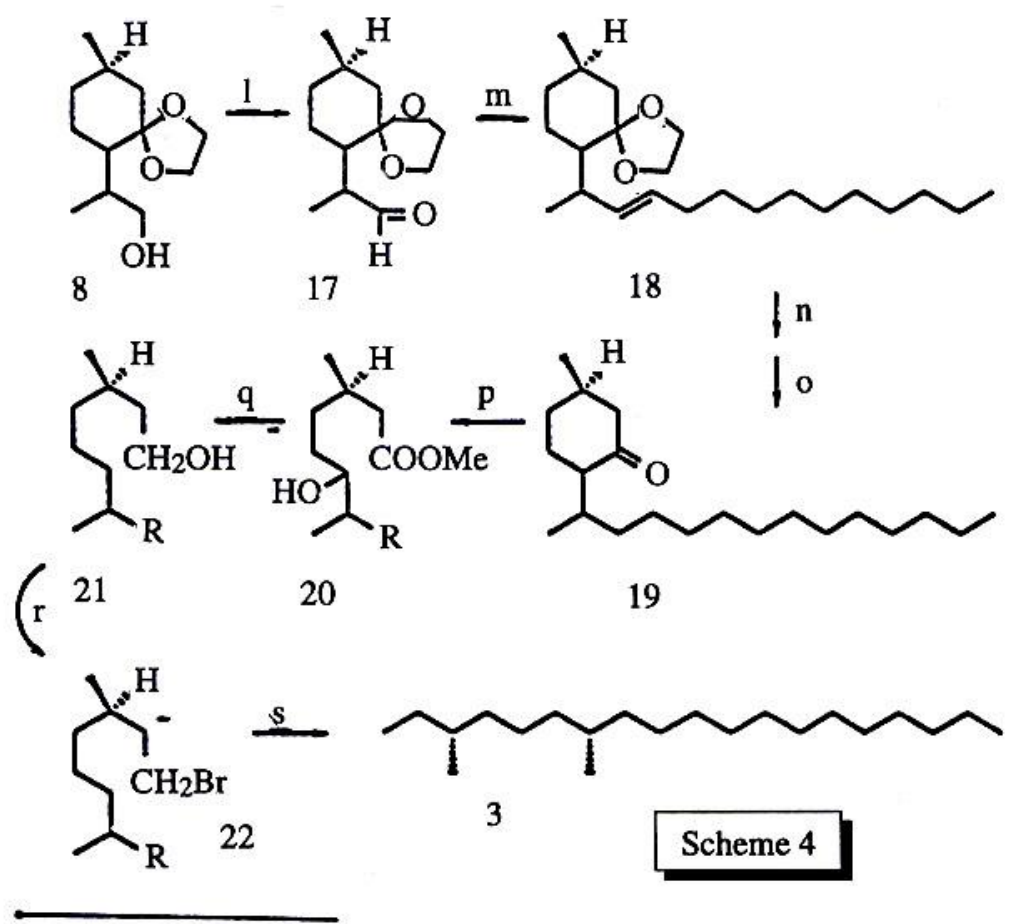

elements of taxodione including the three angularly attached six-membered rings. But even more important, the absolute stereochemistry (the handedness) of the centres identified as C.10 and C.5 is exactly the three dimensional geometry required for "natural" taxodione !

Scheme 5 is a brief outline of the crucial intermediates in our synthesis (Burnell et al. 1987). The white arrows merely draw attention to the part of the molecule that will be modified in the next transformation. The key step was the use of a selenium reagent (Barton $e t$ al. 1981) to oxidise the phenol 25 to the ortho-quinone 26. Some of the reactions were most gratifying. For instance, while conventional wisdom would say such a change was unlikely, compound 26 rearranged spontaneously to 27 and the latter was oxidized to the final taxodione 28 by merely percolating its solution through a column of silica gel. The product was, of course, pure natural taxodione containing none of the 
mirror image form and this was confirmed by measuring the direction and magnitude of its ability to rotate the plane of polarized light. If we had started the synthesis from simple symmetrical chemicals, the final substance would only be half taxodione and half its mirror image and would show no rotation of the plane of light.

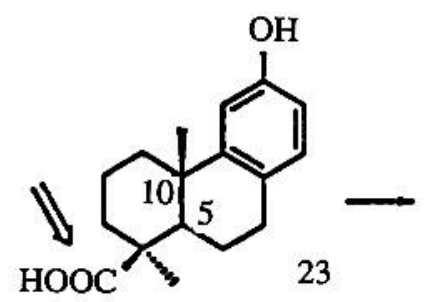

Podocarpic acid<smiles></smiles>

Taxodione 28<smiles>[R20]c1ccc2c(c1)C1(C)CCCC(C)(C)[C@H]1CC2</smiles>

24<smiles>[R20]c1cc2c(cc1C(C)C)CCC1C(C)(C)CCC[C@]21C</smiles>

25

26

Scheme 5

The taxodione preparation was part of a much broader study to corroborate the structures of several naturally occurring aromatic diterpenes by synthesis. Since in all cases we started from podocarpic acid (or the very similar equally affordable dehydro-abietic acid) the final products also possessed the exact stereochemistry (left or right handedness) of the natural substances. Most of the structures proposed in the literature were shown to be correct as in the case of coleon U (Burnell et al. 1981), coleon C 